\title{
Linear Relationships for Modeling CO2 Absorption in Aqueous Alkanolamine Solutions in a Thermodynamically Consistent Way
}

\author{
Xiaoshuai Yuan ${ }^{1}$, Chin Feng $\mathrm{Ng}^{1}$, Nikolic Heather ${ }^{1}$, and Kunlei Liu ${ }^{1}$ \\ ${ }^{1}$ University of Kentucky
}

September 25, 2021

\begin{abstract}
A thermodynamically consistent model for the carbon dioxide (CO2) absorption in aqueous alkanolamine system is of great importance in the research and development of a CO2 capture process. To facilitate the development of thermodynamic models, linear Gibbs free energy, enthalpy, and heat capacity relationships using well-known amines as reference are used to correlate the standard reference state properties of ionic species with those of molecular species in the electrolyte system, which has been approved to provide a reliable and consistent way to estimate required parameters when there is minimal or no appropriate experimental data available. The proposed relationships have been applied to the development of an electrolyte Non-Random Two Liquid (NRTL) activity coefficient model for CO2 absorption in aqueous 1-amino-2-propanol (A2P) solution, as an example to demonstration the methodology. With limited vapor-liquid equilibrium data and other thermodynamic properties, the parameters in the electrolyte NRTL model are identified with good accuracy.
\end{abstract}

\section{Hosted file}

vle_submission.docx available at https://authorea.com/users/437256/articles/539020-linearrelationships-for-modeling-co2-absorption-in-aqueous-alkanolamine-solutions-in-a-

thermodynamically-consistent-way 